TITLE:

\title{
Electric field behavior near a zero- angle contact point in the presence of surface conductivity
}

$\operatorname{AUTHOR}(\mathrm{S})$ :

Techaumnat, B; Hamada, S; Takuma, T

\section{CITATION:}

Techaumnat, B ... [et al]. Electric field behavior near a zero-angle contact point in the presence of surface conductivity. IEEE TRANSACTIONS ON DIELECTRICS AND ELECTRICAL INSULATION 2002, 9(4): 537-543

ISSUE DATE:

2002-08

URL:

http://hdl.handle.net/2433/39979

\section{RIGHT:}

(c)2002 IEEE. Personal use of this material is permitted. However, permission to reprint/republish this material for advertising or promotional purposes or for creating new collective works for resale or redistribution to servers or lists, or to reuse any copyrighted component of this work in other works must be obtained from the IEEE. 


\title{
Electric Field Behavior near a Zero-Angle Contact Point in the Presence of Surface Conductivity
}

\author{
Boonchai Techaumnat \\ Electrical Engineering Department \\ Chulalongkorn University \\ Phyathai Road, Pathumwan \\ Bangkok 10330, Thailand \\ Shoji Hamada and Tadasu Takuma \\ Department of Electrical Engineering \\ Kyoto University, Yoshida-honmachi, Sakyo-ku \\ Kyoto 606-8501, Japan
}

\begin{abstract}
The paper describes the electric field behavior near a contact point in various arrangements with a zero contact angle when surface conductivity is present on a solid surface. Electric field distributions are calculated for arrangements with three contact conditions: point, line, and surface contact. We focus on the effect of surface conductivity on the electric field. It was found that the presence of surface conductivity results in the electric field intensification. Similarly to the effect of volume conductivity, when the surface conductivity is higher than a certain value, a change in the position of the peak electric field takes place. The effect of the surface conductivity is noticeable for as low as $0.8 \mathrm{nS}$.
\end{abstract}

\section{INTRODUCTION}

INSULATION plays an important role in HV electric

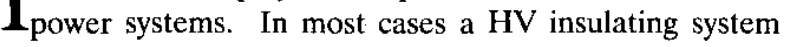
consists of more than one insulating material. For example, a system with a gas or liquid insulating material needs a solid insulator to provide support and separation for a conductor inside. A triple-junction or contact point, the point where three materials (a conductor and two insulating materials) meet each other, exists in such a system. Figure 1 shows an example of contact points occurring due to the presence of a solid spacer in a gas-insulated, coaxial-cylindrical system.

When there exists a contact point in an insulating system, electric field near a contact point often poses a serious problem. The field has a strong effect on insulation design. This is because the electric field near a contact point is often very high. Especially in the case of a system with a gaseous dielectric or vacuum, the dielectric strength of the system substantially decreases when a partial discharge takes place due to field intensification.

Electric field behavior near a contact point has been extensively studied for various conditions [1-4]. It is found that the electric field behavior depends heavily on contact angle and material properties involved. In practice, all di-

Manuscript received on 26 November 2001, in final form 24 April 2002.

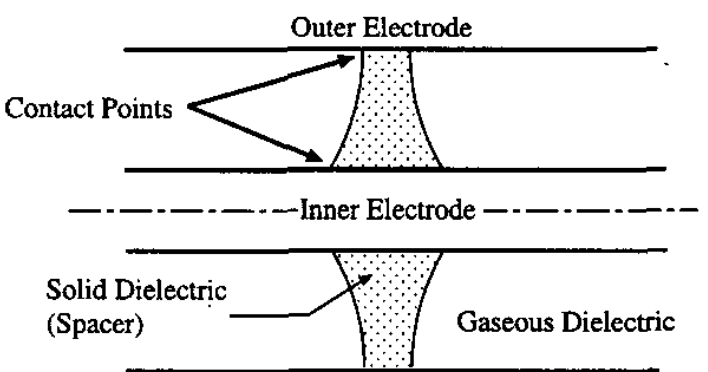

Figure 1. Example of contact points in a gas-insulated coaxial cylinder system.

electrics have a certain degree of volume conductivity, and surface conductivity sometimes exists on a solid dielectric surface due to pollution or humidity. Therefore, the analysis of the electric field in triple-junction problems should be carried out not only for cases of perfect dielectrics, but also for cases of dielectrics with volume conductivity or surface conductivity.

In [5], triple-junction problems were classified into three categories of different electric field behavior, according to their contact angle $\alpha$.

$$
\alpha=90^{\circ}, 0^{\circ}<\alpha<90^{\circ} \text { or } 90^{\circ}<\alpha<180^{\circ} \text { and } \alpha=0^{\circ} \text {. }
$$

The first category was briefly discussed in [5]. Electric field singularity does not take place even though field in- 
tensification occurs in some cases. The second category was completely studied for the effects of permittivity, volume conductivity and surface conductivity. The electric field near a contact point approaches infinity or zero when the materials are perfect dielectrics without any conductivity [3-4]. The effects of volume and surface conductivity were first reported in [5], but not in a complete form. Recently, we have fully analyzed this category of problem in [6]. The presence of constant volume conductivity promotes the field singularity at the contact point, while constant surface conductivity relaxes the field to a uniform distribution near a contact point, and prevents the field from being singular or zero at a contact point.

In the last category, the zero-angle contact condition, the contact condition is further subdivided into line contact (two-dimensional case), point contact (axisymmetrical case), and surface contact. The relationship between the permittivity of a solid dielectric and the electric field behavior near a contact point has been investigated in $[7,8]$. These papers show that the electric field increases with the permittivity of a solid dielectric even on the solid side (except in the configuration of a cylindrical solid dielectric in contact with a grounded plane under a uniform electric field). We have recently found that the effect of the volume conductivity in a solid dielectric is more complicated [9]. Volume conductivity in a solid dielectric promotes the field intensification. Moreover, for point or line contact, the position of the peak electric field shifts from a contact point when the volume conductivity is higher than a certain value, i.e., the peak electric field becomes higher than the electric field at a contact point. For surface contact, the peak electric field usually takes place at a place more or less remote from the contact point even when no conductivity exists and the presence of volume conductivity results in higher peak electric field. However, the effect of surface conductivity on the electric field behavior in this category of problem $\left(\alpha=0^{\circ}\right)$ is still unknown.

In this paper, we describe the effect of surface conductivity on a solid dielectric surface in various arrangements with a zero-angle contact condition. Our aim is to complete the quantitative analysis of the electric field for this contact condition. We numerically calculated the electric field distribution in arrangements (shown later) by using the boundary element method (BEM) with second order curved elements. We focus on the peak electric field on the gaseous side as it is more critical in practice for insulation design.

\section{CALCULATED ARRANGEMENTS}

In order to study the basic behavior of the electric field near a contact point, we chose arrangements I and II as two examples for a triple-junction problem with point or line contact, and arrangements III for a problem with surface contact. All the arrangements are shown in Figure 2. Note that arrangements I and II have different degrees of

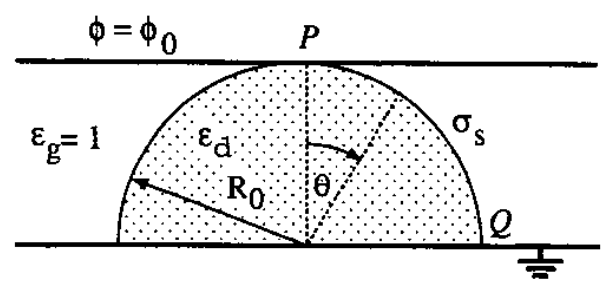

(a)

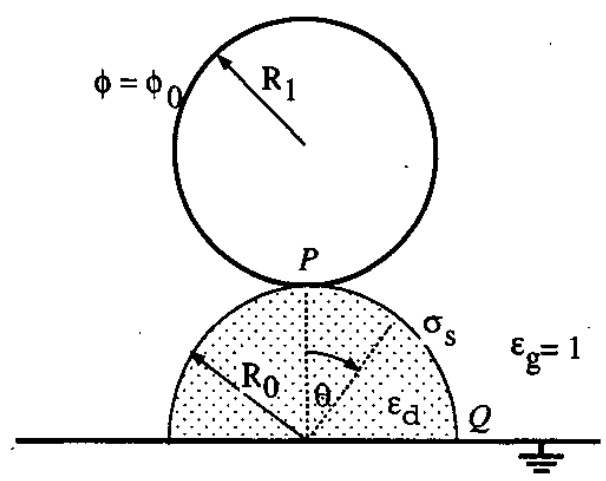

(b)

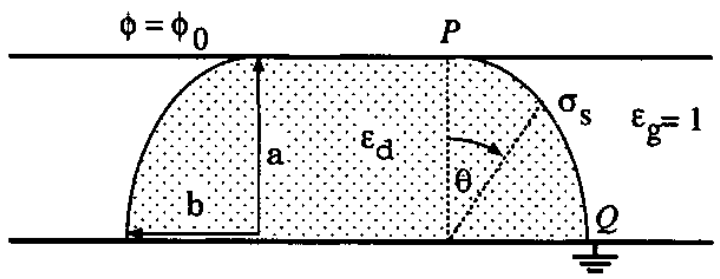

(c)

Figure 2. Arrangements with $\alpha=0^{\circ}$. a, arrangement I; b, arrangement II; c, arrangement III.

the field non-uniformity. Arrangement $I$ is by symmetry equivalent in the electric field to an arrangement of a rounded (cylindrical or spherical) dielectric solid lying between two plane electrodes separated by $2 R_{0}$. Arrangement II is also equivalent to that of a rounded dielectric solid lying between and in contact with two cylindrical or spherical electrodes above and below. The ratio $R_{1} / R_{0}$ of arrangement II is set to 1 in the calculation. The contact conditions of arrangements I and II are line contact in the two-dimensional case and point contact in the axisymmetrical case.

For arrangement III, since the contact condition is surface contact for both the two-dimensional and axisymmetrical cases, we calculated the electric field only for the two-dimensional case. The effect of the radius ratio $b$ to $a$ has been reported in [9]. Here we simply use $b / a=1$ in the calculation.

In this paper we concentrate on the effect of surface conductivity $\sigma_{s}$, and assume that both the gaseous and solid 
IEEE Transactions on Dielectrics and Electrical Insulation

dielectrics in all the arrangements possess no volume conductivity. The relative permittivities $\epsilon_{g}$ of the gaseous dielectric and $\epsilon_{d}$ of the solid dielectric are assumed to be equal to 1 and 4 , respectively, corresponding to the permittivity of the free space and the typical material used for gas-insulated spacers. We have performed the calculation for $\sigma_{s}=0$ to $2.4 \mathrm{nS}$. Although surface conductivity greatly depends on temperature and electric stress, we have chosen a constant value of conductivity to make clear the general characteristics on the effect of conductivity. The conductivity also becomes predominant with decreasing source frequency to dc energization. In this paper the electric field is calculated for $50 \mathrm{~Hz}$ ac. For all the arrangements, $\theta$ represents an angle starting from the contact point $P$ when we describe the field distribution on a rounded dielectric surface.

\section{CALCULATION METHODS}

\subsection{BOUNDARY ELEMENT METHOD FOR A DOMAIN WITH SURFACE CONDUCTIVITY}

As in our work on the calculation of the electric field in triple junction problems [9], we utilize the BEM as the calculation method. The BEM is one of boundary subdivision methods which are generally more accurate in electric field calculation than domain subdivision methods, such as the finite element method and finite difference method.

In the BEM, the electric scalar potential and the normal component of electric field in the outward direction on all the boundary nodes are determined first. Then, the potential $\phi_{p}$ at any point $p$ in the region can be expressed in terms of the potential $\phi$ and the normal component of electric field $E_{n}$ on the boundary $\Gamma$ of the region as

$$
C \phi_{p}=\int_{\Gamma} E_{n} \phi^{*} d \Gamma+\int_{\Gamma} \phi\left(\partial \phi^{*} / \partial n\right) d \Gamma
$$

where $C$ is a coefficient that depends on the position of $p, \phi^{*}$ the fundamental solution of the Poisson equation $\nabla^{2} \phi^{*}=-\Delta^{p}$ ( $\Delta^{p}$ is a Dirac delta function that is everywhere 0 except at $p$ ), and $\left(\partial \phi^{*} / \partial n\right)$ is its normal derivative on the boundary. $C=1$ if $p$ is not on the boundary. If $p$ is on the boundary and the boundary is smooth at $p$, $C=1 / 2$.

In order to calculate the electric field by the BEM in a domain which has surface conductivity inside, we can use the following boundary conditions at an interface with surface conductivity (See Figure 3 ).

$$
\begin{gathered}
\phi_{d}=\phi_{g}, \\
j \omega \epsilon_{d} \epsilon_{0} E_{n d}+j \omega \epsilon_{g} \epsilon_{0} E_{n g}=\nabla_{s} \cdot \vec{J}_{s},
\end{gathered}
$$

where the subscripts $d$ and $g$ denote the solid and gaseous dielectric side, respectively, $\epsilon_{0}$ the permittivity of free
Vol. 9, No. 4; August 2002

539

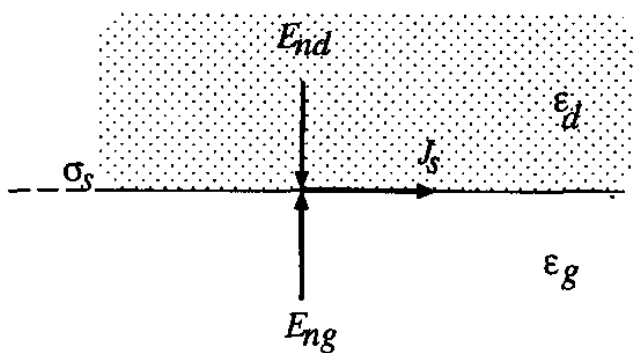

Figure 3. Interface with surface conductivity.

space, $\omega$ the angular frequency, $\nabla_{s} \cdot \vec{J}_{s}$ the tangential divergence or surface divergence possessed by surface current $\vec{J}_{s}$, and $\vec{J}_{s}=-\sigma_{s} \nabla_{s} \phi$. Both the gaseous and solid dielectrics are treated as perfect dielectrics, i.e., lossless media, but the interface has a surface conductivity $\sigma_{s}$. Note that $E_{n d}$ and $E_{n g}$ are defined in the convention of the BEM. $E_{n d}$ and $E_{n g}$ point into the surface from their corresponding region (Figure 3 ), resulting in the plus sign in the left hand side of equation (3). Equation (3) is deduced from the following relations of surface charge density $\rho_{s}$ on the interface.

$$
\begin{gathered}
\nabla_{s} \cdot \overrightarrow{J_{s}}=-\frac{d \rho_{s}}{d t}, \\
\epsilon_{d} \epsilon_{0} E_{n d}+\epsilon_{g} \epsilon_{0} E_{n g}=-\rho_{s} .
\end{gathered}
$$

The plus and minus signs in equation (5) are again in accordance with the directions of $E_{n d}$ and $E_{n g}$ shown in Figure 3.

Directly applying equation (3) to the BEM requires the evaluation of $\nabla_{s} \cdot \nabla_{s} \phi$, resulting in the deterioration of the calculation accuracy. In order to improve the accuracy, the weight residual method with an arbitrary weighting function $w$ can be applied to equation (3) and the following equation is obtained [10].

$$
\int_{\Gamma_{s}} w\left(j \omega \epsilon_{d} \epsilon_{0} E_{n d}+j \omega \epsilon_{g} \epsilon_{0} E_{n g}\right) d \Gamma=-\sigma_{s} \int_{\Gamma_{s}} w \nabla_{s} \cdot \nabla_{s} \phi d \Gamma
$$

where $\Gamma_{s}$ is the interface (boundary) which has surface conductivity $\sigma_{s}$.

Applying the Green's theorem to the above equation yields a boundary condition in which only the tangential divergence operation is required. According to the Green's theorem, the following expression holds for an arbitrary surface $\Gamma$ with a boundary line $L_{\Gamma}$.

$$
\int_{\Gamma} A \frac{\partial B}{\partial x} d \Gamma+\int_{\Gamma} \frac{\partial A}{\partial x} B d \Gamma=\oint_{L_{\Gamma}} A B n_{x} d L
$$

where $A$ and $B$ are arbitrary functions of $(x, y, z)$ in the integration, $x$ the coordinate direction at any arbitrary 
angle with respect to the normal, and $n_{x}$ is the direction cosine between the normal vector $\vec{a}_{n}$ on the boundary and the $x$ direction. Equation (7) represents a relation between the integrals on a surface $\Gamma$ and that on its closed boundary line $L_{\Gamma}$. It is implied from equation (7) that

$$
\int_{\Gamma} A \nabla_{s} \cdot \vec{B} d \Gamma=-\int_{\Gamma} \nabla_{s} A \cdot \vec{B} d \Gamma+\oint_{L_{\Gamma}} A \vec{B} \cdot \vec{a}_{n} d L
$$

We then can rewrite equation (6) as

$$
\begin{aligned}
\int_{\Gamma_{s}} w\left(j \omega \epsilon_{d} \epsilon_{0} E_{n d}+j \omega \epsilon_{g} \epsilon_{0} E_{n g}\right) d \Gamma & \\
= & \sigma_{s} \int_{\Gamma_{s}^{\prime}} \nabla_{s} w \cdot \nabla_{s} \phi d \Gamma-\sigma_{s} \oint_{L_{r_{s}}} w \nabla_{s} \phi \cdot \vec{a}_{n} d L
\end{aligned}
$$

where $L_{\Gamma s}$ is the boundary line of the conduction surface $\Gamma_{s}$, and $\vec{a}_{n}$ is the normal vector on $L_{\Gamma s}$.

Equation (9) represents the boundary condition for the normal component of electric field that we utilize in this paper when surface conductivity exists on an interface.

\subsection{COMPARISON WITH ANALYTICAL EXPRESSION}

In order to estimate the accuracy of the numerical method, we calculated the electric field for a configuration in Figure 4. The configuration consists of a dielectric sphere in a uniform electric field $E_{0}$. The sphere has a conductivity $\sigma_{s}$ on its surface. The other calculation conditions are: $R=1 \mathrm{~m}, E_{0}=1 \mathrm{~V} / \mathrm{m}, \epsilon_{g}=1, \epsilon_{d}=4$, and $\sigma_{s}=10$ or $100 \mathrm{nS}$.

Figure 5 compares the calculation results by the BEM with the results calculated from the following analytical expressions of the potential in Reference [11]

$$
\begin{array}{r}
\dot{\phi}_{g}=-E_{0} \cos \theta\left[r-\frac{1}{1+T^{2}}\left(1+\frac{\epsilon_{d}-\epsilon_{g}}{2 \epsilon_{g}+\epsilon_{d}} T^{2}\right) \frac{R^{3}}{r^{2}}\right. \\
\left.+j \frac{3 \epsilon_{g}}{2 \epsilon_{g}+\epsilon_{d}} \frac{T}{1+T^{2}} \frac{R^{3}}{r^{2}}\right],
\end{array}
$$

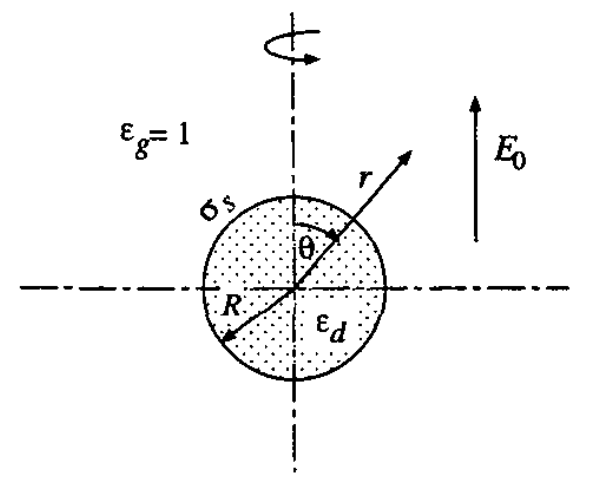

Figure 4. Configuration for accuracy estimation.

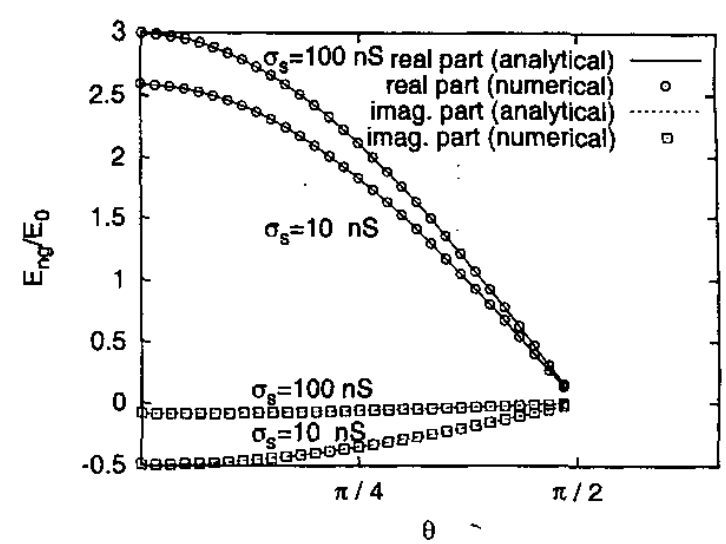

Figure 5. Normal components of the electric field on the sphere surface.

$$
\phi_{d}=-\frac{3 \epsilon_{g}}{2 \epsilon_{g}+\epsilon_{d}} \frac{T}{1+T^{2}}(T+j) r E_{0} \cos \theta
$$

where

$$
T=\omega R\left(2 \epsilon_{g}+\epsilon_{d}\right) / 2 \sigma_{s}
$$

In Figure 5, the abscissa is the angle $\theta$ shown in Figure 4 , and the ordinates are the real and imaginary parts of the normal component of the electric field on the gaseous side of the solid surface. The calculation was performed by the technique described in the previous section with a subdivision of 30 elements. Figure 5 shows a very good agreement between the numerical and analytical results. The errors of calculation results are not shown in Figure 5 , but the maximum error of the electric field is less than $0.0004 \%$ on the solid surface. This confirms the validity and accuracy of our calculation method. However, it is to be noted that much more fine subdivision is required in order to obtain an accurate result in the proximity of a contact point in arrangements I, II, and III since the field is highly non-uniform near a contact point.

\section{RESULTS AND DISCUSSIONS}

Our calculation in [9] shows that the tangential electric field rapidly decreases to zero along the solid surface near a contact point. From equation (3) we expect that the presence of $\sigma_{s}$ alters the electric field particularly near a contact point. In this section, first we examine the effect of surface conductivity $\sigma_{s}$ starting from an electric field distribution when the conductivity is low. Next, we will discuss the electric field behavior in arrangements with a line, point, and surface contact.

In the following sections, we normalize the electric field in arrangements by $E_{c 1}$, the contact-point electric field strength when $\epsilon_{d}=1$ in the perfect dielectric condition $\left(\sigma_{s}=0\right)$. So the field intensification caused by surface conductivity can be readily understood. The values of $E_{c 1}$ for all the arrangements are listed in Table 1. 
Table 1. Value of $E_{c 1}$ for each arrangement.

\begin{tabular}{lcc}
\hline & \multicolumn{2}{c}{$E_{c 1} / \phi_{0}\left[\mathrm{~m}^{-1}\right]$} \\
\cline { 2 - 3 } Arrangement & Two-Dimensional Case & Axisymmetrical Case \\
\hline I & 1.000 & 1.000 \\
II & 1.315 & 1.770 \\
III & 1.000 & 1.000 \\
\hline
\end{tabular}

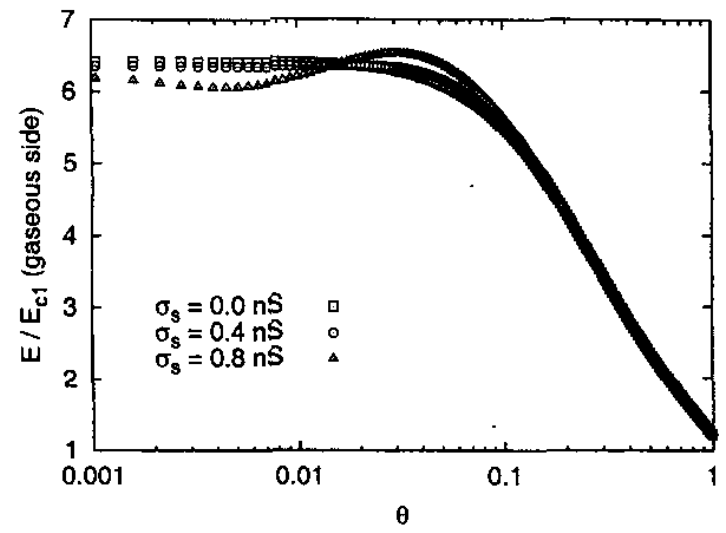

(a)

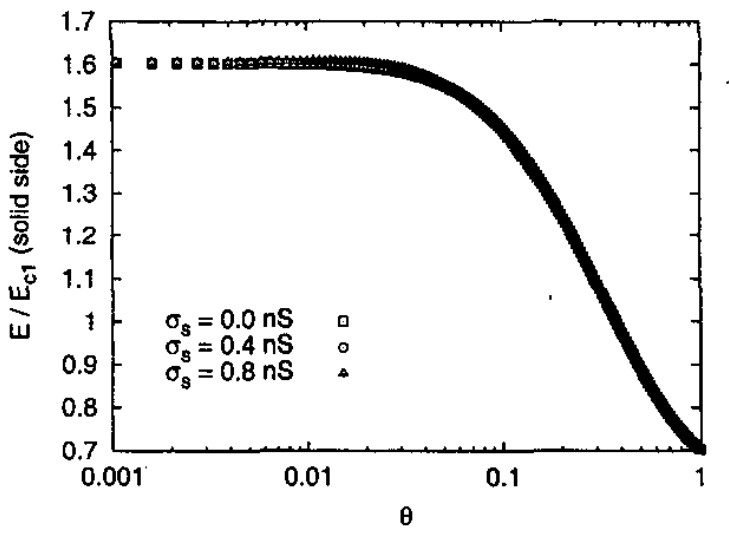

(b)

Figure 6. Magnitude of the electric field along the solid dielectric surface in arrangement I when surface conductivity is present $\left(\epsilon_{d}=\right.$ 4). a, on gaseous dielectric side; b, on solid dielectric side.

\subsection{EFFECT OF $\sigma_{S}$ ON ELECTRIC FIELD DISTRIBUTION}

Figure 6 shows the magnitude of the electric field along the solid surface in arrangement $I$ in the two-dimensional case when $\sigma_{s}$ is small. We present the abscissa in a logarithmic scale so as to more clearly observe the field near a contact point. Each electric field in the ordinate is normalized by $E_{c 1}$. As can be seen from Figure 6, the presence of $\sigma_{s}$ affects the electric field more on the gaseous dielectric side than on the solid dielectric side. The shift of the peak electric field occurs at $\sigma_{s}=0.8 \mathrm{nS}$. This is very similar to the effect of volume conductivity in arrangements with zero contact angle [9]. Figure 7 shows the tangential electric field $E_{t}$ along the solid surface. It is obvious that the tangential field is almost the same for this

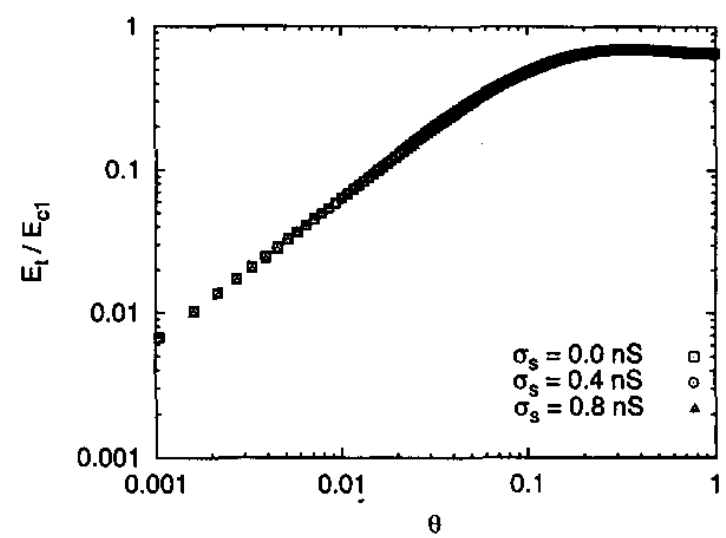

(a)

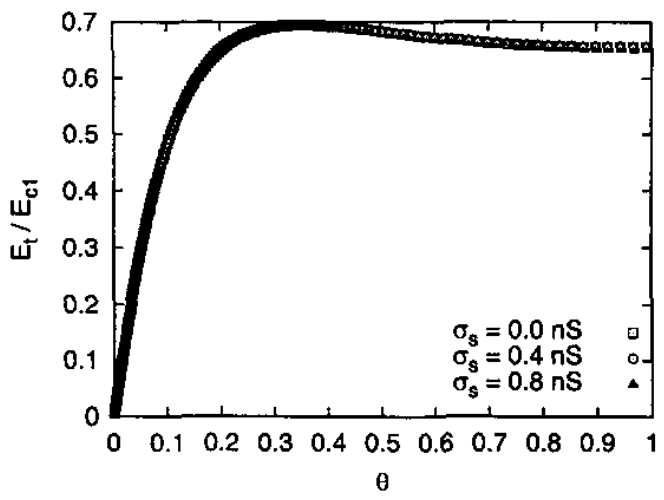

(b)

Figure 7. Tangential electric field along the solid dielectric surface in arrangement I. a, logarithmic scale; $b$, linear scale.

range of $\sigma_{s}(0$ to $0.8 \mathrm{nS})$. This implies that the change in the electric field distributions in Figure 6(a) is mainly caused by the change in the normal component of electric field.

The reason why the peak electric field position changes is considered as follows. The effect of $\sigma_{s}$ is expressed in Equation (3) by the term $\nabla_{s} \cdot \vec{J}$, i.e., $\sigma_{s} \nabla_{s} \cdot \overrightarrow{E_{t}}$ and Figure $7 \mathrm{~b}$ shows that $\left|\nabla_{s} \cdot \vec{E}_{t}\right|$ is very high near the contact point (since $\left.\nabla_{s} \cdot \vec{E}_{t} \propto d E_{t} / d \theta\right)$. If there is no surface conductivity $\left(\sigma_{s}=0\right)$ and $\sigma_{d}=\sigma_{g}=0$, only the terms $j \omega \epsilon_{d} \epsilon_{0} E_{n d}$ and $j \omega \epsilon_{g} \epsilon_{0} E_{n g}$ exist in equation (3) and $\left|j \omega \epsilon_{d} \epsilon_{0} E_{n d}\right|$ $=\left|j \omega \epsilon_{g} \epsilon_{0} E_{n g}\right|$. This means that the normal components of the displacement current in both sides are of the same magnitude. The distribution of $j \omega \epsilon E_{n}$ is very similar to that of $E / E_{c 1}$ for $\sigma_{s}=0 \mathrm{nS}$ in Figure 6. Near a contact point the current density gradually increases with decreasing distance from a contact point and reaches its peak at a contact point. Figure 8 compares the normal component of the displacement current density $j \omega \epsilon E_{n}$ on the solid dielectric surface for $\sigma_{s}=0.8 \mathrm{nS}$. It can be seen that near the contact point the real part of $j \omega \epsilon E_{n}$ in the gaseous side, $\operatorname{Re}\left\{j \omega \epsilon_{g} \epsilon_{0} E_{n g}\right\}$ increases, but the imaginary part $\operatorname{Im}\left\{j \omega \epsilon_{g} \epsilon_{0} E_{n g}\right\}$ decreases when compared with those in 


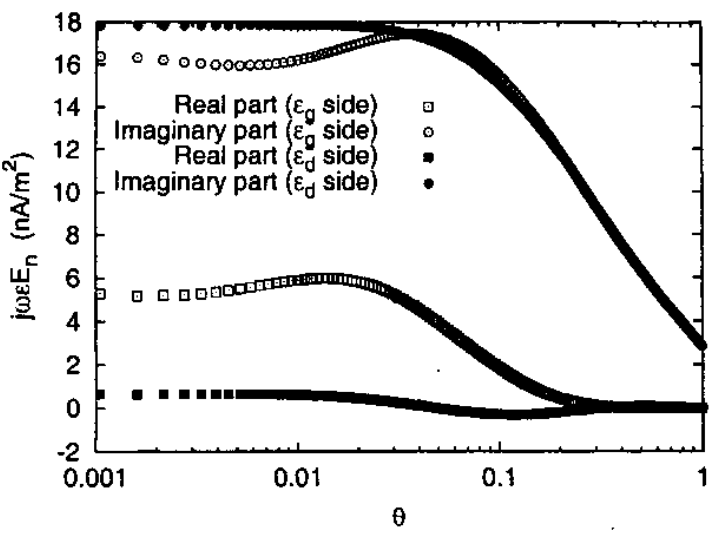

Figure 8. Normal components of the displacement current $\left(j \omega \epsilon E_{n}\right)$ along the solid dielectric surface in arrangement $I$. Note that the real part of $j \omega \epsilon E_{n}$ corresponds to the imaginary part of $E_{n}$.

the solid side. The decrease of $\operatorname{Im}\left\{j \omega \epsilon_{g} \epsilon_{0} E_{n g}\right\}$ results in its peak position at a point remote from a contact point. As a consequence, the peak position of $j \omega \epsilon_{g} \epsilon_{0} E_{n g}$ is at more or less the same position because the imaginary part is predominant over the real part. The normal component of the electric field $E_{n g}$ is explicitly proportional to $j \omega \epsilon E_{n g}$ but $\pi / 2$ out of phase. Therefore, the field distribution is similar to that of the current density and has its peak remote from a contact point. The peak position of the imaginary part of $j \omega \epsilon_{g} \epsilon_{0} E_{n g}$ is approximately at $\theta=0.04$ in Figure 8 which is close to the peak position of the field for $\sigma_{s}=0.8 \mathrm{nS}$ in Figure 6a.

\subsection{PEAK ELECTRIC FIELD ON SOLID SURFACE}

In this section, we investigate the effect of $\sigma_{s}$ on the peak electric field when the conductivity is higher than that in the previous section. As an example of the electric field distribution, Figure 9 shows the normalized electric field magnitude along the solid surface in arrangement $\mathbf{I}$. Figures $9 \mathrm{a}$ and $9 \mathrm{~b}$ give the calculation results in the twodimensional and axisymmetrical cases, respectively. As can be seen in the figures, the presence of surface conductivity results in the electric field intensification near the contact point. The effect of $\sigma_{s}$ is noticeable even when $\sigma_{s}$ is as low as $0.8 \mathrm{nS}$, and a relatively small $\sigma_{s}$ (about 1.6 or 2.4 $\mathrm{nS}$ ) results in a substantially higher peak field strength than that in the case where no surface conductivity exists. The electric field on the solid surface is maximal at a point remote from the contact point when $\sigma_{s}$ is higher than a certain value as explained in the previous section. With a further increase of $\sigma_{s}$, the position of the peak field strength then moves closer to the contact point. This is very important because the higher field takes place at a smaller gap between the solid surface and the electrode. The electric field strength is higher in the axisymmetrical case than in the two-dimensional case.

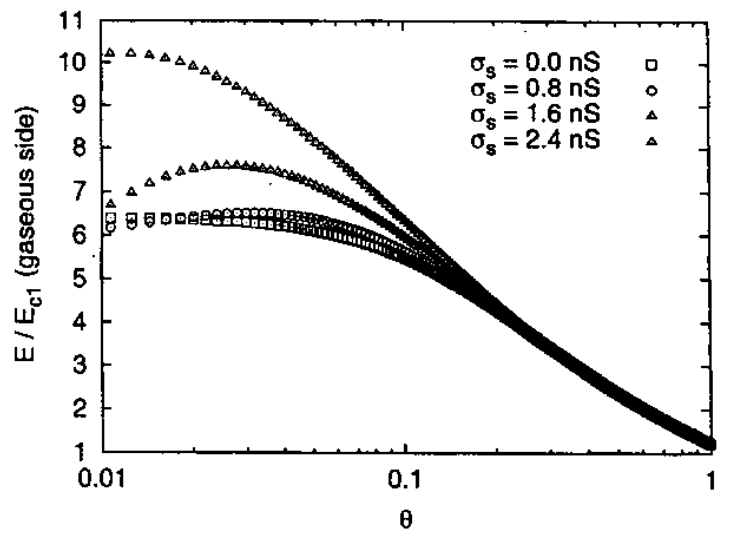

(a)

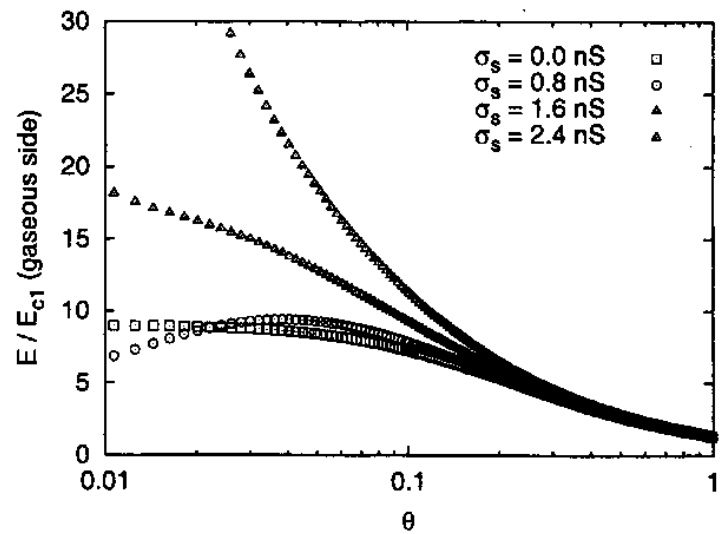

(b)

Figure 9. Magnitude of the electric field along the solid dielectric surface in arrangement I. a, two-dimensional case; b, axisymmetrical case.

Figure 10 presents the magnitude of the peak electric field in' relation to the surface conductivity. Obviously, when the surface conductivity becomes predominant near a contact point, the electric field is much more intensified. The effect of $\sigma_{s}$ is higher in arrangement II in which the electric field is basically more non-uniform than that in arrangement $I$. The variations of the peak field strength in the two-dimensional and axisymmetrical cases are similar.

Figure 11 displays the magnitude of the peak electric field in arrangement III in the two-dimensional case. If we compare the peak field strength with that in arrangement $I$ in the two-dimensional case (Figure 10a) which has the same $E_{c 1}$, the relation between the peak field strength and $\sigma_{s}$ is similar in both cases, except that the field is lower in arrangement III (surface contact).

Obviously from the results, the presence of $\sigma_{s}$ intensifies the electric field in arrangements of a zero contact angle, whether the contact condition is line, point, or surface contact. While it is reported that $\sigma_{s}$ moderates the electric field in arrangements of a contact angle $0^{\circ}<\alpha<$ 


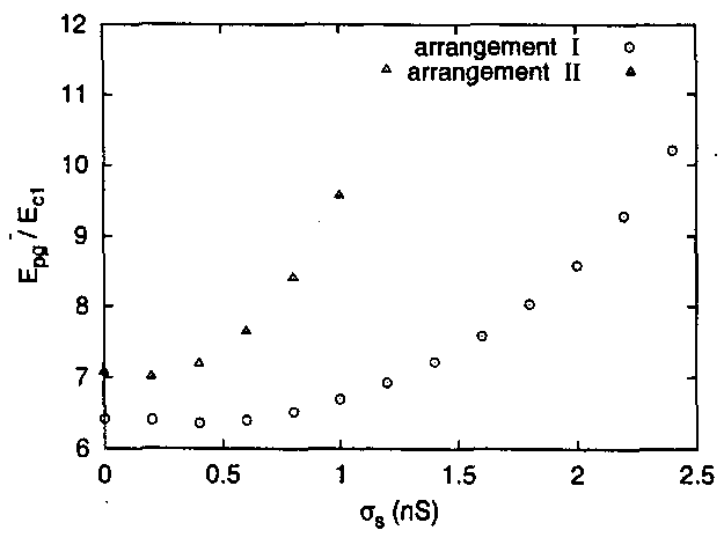

(a)

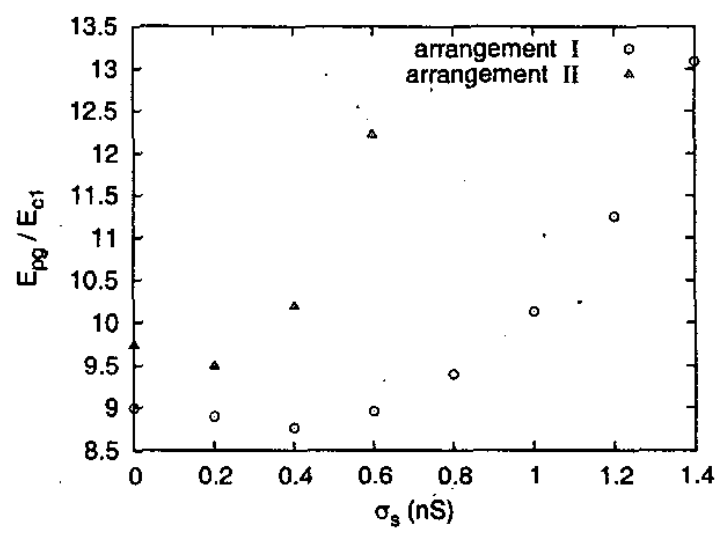

(b)

Figure 10. Magnitude of the peak electric field in arrangements I and II. a, two-dimensional case; b, axisymmetrical case.

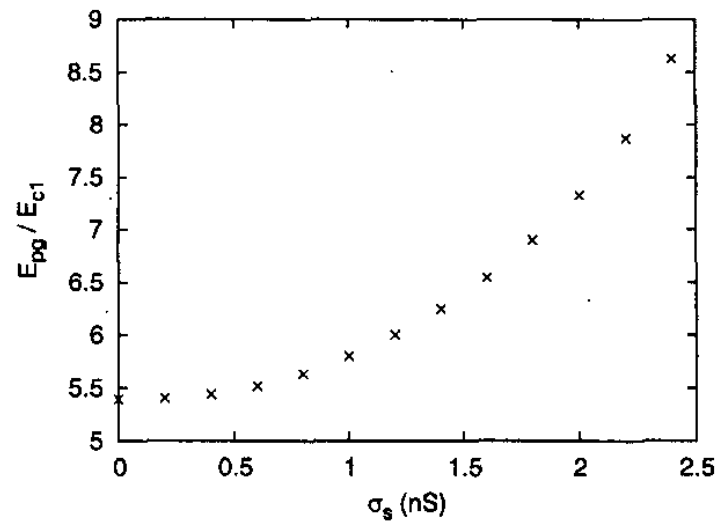

Figure 11. Magnitude of the peak electric field in arrangement III.

$90^{\circ}$ or $90^{\circ}<\alpha<180^{\circ}$ in [4], this paper shows that $\sigma_{s}$ has an opposite effect for the zero-angle contact condition.

\section{CONCLUSIONS}

THE effect of surface conductivity $\sigma_{s}$ on the electric field in a triple-junction problem with a zero contact angle has been investigated for the contact conditions of point, line, and surface contacts. The effect is totally different from that in a problem with a finite angle $0^{\circ}<\alpha<$ $90^{\circ}$ or $90^{\circ}<\alpha<180^{\circ}$. The presence of surface conductivity promotes the electric field intensification near a contact point, particularly in the gaseous dielectric side. Similarly to the case of volume conductivity, the peak electric field does not take place at a contact point when $\sigma_{s}$ is greater than a certain value. We have found that the effect of surface conductivity is considerably high even with a small value of conductivity (about $0.8 \mathrm{nS}$ in our calculation). The field intensification due to the surface conductivity is highest in the point contact and lowest in the surface contact.

\section{REFERENCES}

[1] J. Meixner, "The Behavior of Electromagnetic Fields at Edges", IEEE Trans. Antennas and Propagation, Vol. 20, pp. 442-446, 1972.

[2] R. Mittra and S. W. Lee, Analytical Techniques in the Theory of Guided Waves, Macmillan, New York, pp. 4-11, 1971.

[3] T. Takuma, T. Kouno, and H. Matsuda, "Field Behavior Near Singular Points in Composite Dielectric Arrangements", IEEE Trans. EI, Vol. 13, pp. 426-435, 1978.

[4] T. Takuma, T. Kawamoto, and H. Fujinami, "Effect of Conduction on Field Behavior Near Singular Points in Composite Medium Arrangements", IEEE Trans. EI, Vol. 17, pp. 269-275, 1982.

[5] T. Takuma, "Field Behavior at a Triple Junction in Composite Dielectric Arrangements", IEEE Trans. EI, Vol. 26, pp. 500-509, 1991.

[6] B. Techaumnat, S. Hamada, and T. Takuma, "Effect of Conductivity on Electric Ficld Behavior Near a Contact Point", 12th ISH, Vol. 1, No. 1-2, 2001.

[7] T. Takuma and T. Kawamoto, "Field Intensification Near Various Points of Contact With a Zero Contact Angle Between a Solid Dielectric and an Electrode", IEEE Trans. PAS., Vol. 103, pp. 2486-2494, 1984.

[8] T. Takuma and T. Kawamoto, "Electric Field at Various Points of Contact Between Rounded Dielectric and Electrode",5th ISH No. 33.09, 1987.

[9] B. Techaumnat, S. Hamada, and T. Takuma, "Electric Field Behavior Near a Contact Point in the Presence of Volume Conductivity", IEEE Trans. DEI, Vol. 8, pp. 930-935, 2001.

[10] A. Nicolas, J. Rasolonjanahary, and L. Krahenbuhl, "Computation of Electric Fields and Potential on Polluted Insulators Using a Boundary Element Method", IEEE Trans. MAG., Vol. 28, pp. $1473-1476,1992$.

[11] T. Takuma, T. Kouno, and H. Matsuda, "Field Behavior Near Singular Points in Composite Dielectric Arrangements", IEEE Trans. EI, Vol. 13, pp. 426-435, 1978. 